\title{
Expression and Localization Patterns of a Small Heat Shock Protein that Interacts with the Helicase Domain of Cucumber Green Mottle Mosaic Virus
}

\author{
Shanshan Liu, ${ }^{1}$ Lifeng Liu, ${ }^{1}$ Miguel A. Aranda, ${ }^{2}$ Bin Peng, ${ }^{1}$ and Qinsheng Gu${ }^{1, \dagger}$ \\ ${ }^{1}$ Henan Provincial Key Laboratory of Fruit and Cucurbit Biology, Zhengzhou Fruit Research Institute, Chinese Academy of Agricultural \\ Sciences, Zhengzhou 450009, Henan, China \\ ${ }^{2}$ Centro de Edafología y Biología Aplicada del Segura-CSIC, 30100 Espinardo, Murcia, Spain \\ Accepted for publication 24 April 2019.
}

\begin{abstract}
Cucumber green mottle mosaic virus (CGMMV), a member of the genus Tobamovirus (family Virgaviridae), is an economically important virus that has detrimental effects on cucurbit crops worldwide. Understanding the interaction between host factors and CGMMV viral proteins will facilitate the design of new strategies for disease control. In this study, a yeast two-hybrid assay revealed that the CGMMV helicase (HEL) domain interacts with a Citrullus lanatus small heat shock protein (sHSP), and we verified this observation by performing in vitro GST pulldown and in vivo coimmunoprecipitation assays. Measurement of the levels of accumulated sHSP transcript revealed that $s H S P$ is upregulated on initial CGMMV infection in both Nicotiana benthamiana and

C. lanatus plants, although not in the systemically infected leaves. We also found that the subcellular localization of the sHSP was altered after CGMMV infection. To further validate the role of sHSP in CGMMV infection, we produced and assayed $N$. benthamiana transgenic plants with up- and down-regulated sHSP expression. Overexpression of sHSP inhibited viral RNA accumulation and retarded disease development, whereas sHSP silencing had no marked effect on CGMMV infection. Therefore, we postulate that the identified sHSP may be one of the factors modulating host defense mechanisms in response to CGMMV infection and that the HEL domain interaction may inhibit this sHSP function to promote viral infection.
\end{abstract}

Cucumber green mottle mosaic virus (CGMMV) is an economically important virus that causes severe losses in cucurbit crops worldwide (Reingold et al. 2016). Cucurbit plants infected by CGMMV exhibit green mottling and mosaic on their leaves, and their fruits are unmarketable because of malformations, flesh sponginess, rotting, yellowing, and dirty red discoloration (Dombrovsky et al. 2017). Understanding the interaction between host factors and CGMMV viral proteins would contribute to the design of new strategies for the control of this disease.

As obligate parasites with very small genomes, plant pathogenic viruses have evolved complex interactive networks with their hosts to complete their lifecycles. For the virus to achieve successful infection, it is necessary that the interactions between viral and host factors proceed in a specific manner. This includes viral genome amplification, cell-to-cell and systemic movement, disease symptom development, and defense against host antiviral responses (García and Pallás 2015; Whitham and Wang 2004). Characterizing the specific interactions between viral and host proteins can shed light on how viruses utilize and alter cellular resources and how hosts respond to virus invasion.

CGMMV, a member of the genus Tobamovirus in the family Virgaviridae, possesses a 6,423-nucleotide single-stranded positivesense RNA genome with four open reading frames (ORFs) encoding four proteins. The two coterminal $5^{\prime}$ proximal ORFs encode the

${ }^{\dagger}$ Corresponding author: Q. Gu; guqinsheng@ caas.cn

Funding: This work was jointly funded by Modern Agro-industry Technology Research System grant CARS-26-13 and Agricultural Science and Technology Innovation Program grant CAAS-ASTIP-2018-ZFRI. M. A. Aranda was supported by grant 2I16SA000057 from Región de Murcia, Consejería de Empleo Universidades y Empresa, RIS3MUR Program, Spain.

*The $\boldsymbol{e}$-Xtra logo stands for "electronic extra" and indicates that one supplementary table is published online.

The author(s) declare no conflict of interest.

(c) 2019 The American Phytopathological Society
129- and 186-kDa proteins required for efficient viral replication. Both replicase proteins possess an $\mathrm{N}$-terminal methyltransferase (MT) domain and a 129-kDa C-terminal helicase (HEL) domain that are separated by a unique intervening region (IR) (Ishibashi and Ishikawa 2016; Rozanov et al. 1992). The longer 186-kDa replicase is expressed by suppression of a UAG termination codon and encodes an RNA-dependent RNA polymerase (RdRp) motif at its C-terminal domain (Beier et al. 1984). During replication, CGMMV produces two 3' coterminal subgenomic messenger RNAs (mRNAs) that are translated into two additional proteins, a cell-to-cell movement protein and a coat protein (Ugaki et al. 1991).

The CGMMV HEL domain is one of the domains shared by both the 129- and 186-kDa proteins. HEL domains play an important role in unwinding the double-stranded helical structure of nucleic acids by using the free energy of NTP hydrolysis. They are ubiquitous in many organisms, including viruses, and they are involved in all aspects of nucleic acid metabolism, including gene replication, transcription, translation, repair, and recombination (Abdelhaleem 2009). HEL domains have been classified into six superfamilies (SFs; SF1 to SF6) according to their motifs, amino acid sequence, and spacing (Gorbalenya and Koonin 1993; Singleton et al. 2007). The tobamovirus HEL domain belongs to the SF1 group and interacts with host proteins associated with host defense responses, viral symptom development, and replication (Goregaoker and Culver 2003; Ishibashi et al. 2010). Tobacco mosaic virus (TMV) suppresses salicylic acid (SA)-mediated host basal defenses through the interaction between its HEL domain and the host NAC domain transcription factor ATAF2 (Wang et al. 2009). The HEL domains of TMV and tobacco etch virus have been found to interact with a plant ortholog of P58IPK, and this interaction has been proposed to activate the P58 $8^{\mathrm{IPK}}$ ortholog to enhance virus multiplication by preventing PKR-mediated translational suppression and apoptosis (Bilgin et al. 2003; Goodman et al. 2009). The TMV HEL domain has also been found to interact with the host AUX/IAA proteins, and it suppresses their function to avoid host age-related resistance and consequently, enhance TMV infection (Padmanabhan et al. 2008). Furthermore, by interacting with the $\mathrm{N}$ protein in an ATP-dependent manner, the TMV HEL domain also 
functions as an elicitor of $\mathrm{N}$-mediated resistance (Les Erickson et al. 1999; Ueda et al. 2006).

Tomato Tm-1, which confers resistance to tobamoviruses, interacts with the HEL domain of tomato mosaic virus (ToMV), although not with that of a ToMV mutant that can overcome tomato resistance, suggesting that Tm-1 inhibits ToMV replication by binding to the viral HEL domain (Ishibashi et al. 2007). TOM1, which attaches to the tobamovirus replication complex by interacting with the viral HEL domain, facilitates the tethering of viral replication proteins to membranes. Knockout of TOM1 and its homolog TOM3 in Arabidopsis thaliana and Nicotiana tabacum inhibits tobamovirus multiplication, suggesting that TOM1 plays an important role in viral replication by interacting with the HEL domain (Asano et al. 2005; Fujisaki et al. 2006). The foregoing observations accordingly indicate that, by interacting with certain host factors, the HEL domain plays multiple roles in tobamovirus infection.

Plant heat shock proteins (HSPs) are ubiquitous proteins present in plant cells. They are stress-related proteins that participate in a broad range of cellular biological processes, including the modulation of cellular immune responses and the mediation of substrate-protein folding, refolding, oligomeric assembly, translocation, and degradation (Hartl et al. 2011). The small heat shock protein (sHSP) family is one of the most abundant and complex subsets among the five known classes of HSPs (HSPp100/ClpB, HSP90, HSP70/DnaK, HSP60/GroEL, and sHSP), and it is characterized by a conserved $\alpha$-crystallin domain from amino acids 80 to 100 in its C-terminal region (Haslbeck et al. 2005; Wang et al. 2004). The monomeric size of sHSPs ranges between 12 and $42 \mathrm{kDa}$; however, in most cases, sHSPs form large oligomers containing 8 to 32 subunits, which show higher substrate affinity under stress and bind to nonnative proteins of similar weight (Basha et al. 2010; Tyedmers et al. 2010). The key function of sHSPs is to prevent nonnative protein aggregation, and in this regard, they can create a transient reservoir of substrates by forming a soluble complex with nonnative proteins for their subsequent refolding by ATP-dependent chaperone systems (Lee et al. 1997; Mchaourab et al. 2009). Furthermore, it is well known that sHSPs can stabilize several intracellular proteins, including defense-related signaling components, through their chaperone activity (Ahmed et al. 2015). In response to stress, the sHSPs interact with unfolded RNA binding proteins and their protected mRNAs, forming heat shock granules in the cell cytoplasm (Nover et al. 1989). sHSPs are commonly associated with abiotic stress conditions, such as heat, drought, cold, heavy metal, and oxidative stress; however, some plant sHSPs have been reported to play a role in defense against infection by fungi, bacteria, and nematodes (Ahmed et al. 2015; Escobar et al. 2003; Maimbo et al. 2007). Although a number of previous studies have reported on the involvement of plant HSP70s and HSP90s in viral infection (Mine et al. 2012), there have been very few such studies on sHSPs. sHSPs have, nevertheless, been shown to be induced in response to infection by diverse RNA viruses in susceptible A. thaliana plants (Whitham et al. 2003). Similarly, the interaction between rice stripe virus (RSV) $\mathrm{Pc} 4$ protein and a rice sHSP may be necessary for maintaining a denatured Pc4 competent for transport through the plasmodesmata (Lu et al. 2009). Furthermore, the RSV RdRp protein has been shown to interact with an sHSP, thereby altering the expression pattern and subcellular distribution of the sHSP (Li et al. 2015).

Although at present, the identity of the host factors that interact with CGMMV proteins remains unknown, in this study, we identified an interaction between the HEL domain of CGMMV and a host sHSP, and we found that expression of the sHSP is significantly upregulated on initial CGMMV infection. We also showed that overexpression of Nicotiana benthamiana small heat shock protein (NbsHSP) in transgenic $N$. benthamiana inhibits CGMMV accumulation and delays symptom development, which provides compelling evidence that the interaction between the
CGMMV HEL domain and the host sHSP may promote viral infection by inhibiting host sHSP function in defense responses.

\section{MATERIALS AND METHODS}

Plants and virus inoculation. All plants used in this study were grown in $10-\mathrm{cm}$ pots filled with a mixture of meadow soil and vermiculite and maintained in growth chambers at $26^{\circ} \mathrm{C}$ under a 16/8-h light/dark regime and $70 \%$ RH. The CGMMV infectious clone C284R used in this study was constructed in our laboratory (Liu et al. 2017). N. benthamiana plants at the eight-leaf stage and Citrullus lanatus 'Zhongke 6' plants at the expanded cotyledon stage were infected with C284R via the genus Agrobacteriummediated inoculation as described previously (Liu et al. 2017). The mock plants were similarly infected via the genus Agrobacteriummediated inoculation using the empty vector used in constructing the infectious clone C284R. For mechanical inoculation, $1 \mathrm{~g}$ of fresh $N$. benthamiana leaves infected with CGMMV were ground in $10 \mathrm{ml}$ of inoculation buffer. Approximately 4-week-old wild-type (WT) or transgenic $N$. benthamiana plants were inoculated mechanically and then maintained at $26^{\circ} \mathrm{C}$. Mock plants were inoculated mechanically with a homogenate of healthy $N$. benthamiana leaves. At sampling time, leaves were collected from the plants, frozen, and stored at $-80^{\circ} \mathrm{C}$ until use.

Complementary DNA library construction. A C. lanatus leaf-specific complementary DNA (cDNA) library was constructed as pray from $C$. lanatus leaves infected with CGMMV following the procedures outlined in the Make Your Own "Mate \& Plate" Library System User Manual (Clontech). A CGMMV cDNA fragment (nucleotides 2,635 to 3,399] encoding the full-length HEL domain was amplified using $2 \times$ Fast Pfu Master Mix (Novoprotein) and then inserted into the SmaI site of a yeast GAL4 binding domain pGBKT7 (BD) vector using an In-Fusion HD Cloning Kit (Takara) to create the recombinant bait plasmid BD-HEL. The plasmids BD-129, BD-MT, and BD-IR were constructed using the same procedure as used for BD-HEL. The coding sequences of $\mathrm{ClsHSP}$ and $N b s H S P$ were amplified from $C$. lanatus and $N$. benthamiana cDNA using the primer pairs AD-ClsHSP-F/AD-ClsHSP-R and AD-NbsHSP-F/AD-NbsHSP-R, respectively, and then, they were inserted into the SmaI site of a yeast GAL4 transcriptional activation domain (AD) pGADT7 vector using the In-Fusion HD Cloning Kit to create the recombinant pray plasmids AD-ClsHSP and AD-NbsHSP.

Yeast transformation and library screening. Yeast transformation and library screening were conducted in accordance with the Yeastmaker Yeast Transformation System 2 and Matchmaker Gold Yeast Two-Hybrid System User Manual (Clontech). The positive candidate plasmids containing the $C$. lanatus cDNAs were isolated and sequenced. To confirm the protein-protein interactions in yeast, the bait and pray plasmids were cotransformed into Saccharomyces cerevisiae strain Y2HGold (Gietz and Woods 2002). Cotransformants were initially plated on synthetic defined medium lacking leucine and tryptophan with $40 \mu \mathrm{g} / \mathrm{ml} \mathrm{X- \alpha -Gal}$ (SD/-Leu/-Trp/X- $\alpha$-Gal) medium, and positive yeast colonies were subsequently transferred onto higher-stringency SD/-Ade/-His/Leu/-Trp/X- $\alpha$-Gal medium. Meanwhile, AD-T and BD-53 were cotransformed as a positive control, and AD-T and BD-Lam were cotransformed as a negative control. Three independent experiments were performed to confirm these results.

In vitro pull-down assay. For the in vitro pull-down assay, the coding sequence of the HEL domain was amplified with the primer pair PD-HEL-F/PD-HEL-R using the plasmid BD-HEL as a template and then inserted into the SmaI site of a pGEX-6p-1vector using the In-Fusion HD Cloning Kit to create the recombinant plasmid pGEX-6p-HEL. Similarly, the coding sequences of $C l s H S P$ and $N b s H S P$ were amplified with the primer pairs PDClsHSP-F/PD-ClsHSP-R or PD-NbsHSP-F/PD-NbsHSP-R using the plasmid AD-ClsHSP or AD-NbsHSP as a template, respectively, 
and then, they were inserted into the $\mathrm{NcoI} / \mathrm{XhoI}$ sites of a pET-28a vector using the In-Fusion HD Cloning Kit to create the recombinant plasmids pET-28a-ClsHSP and pET-28a-NbsHSP. The recombinant plasmids were transformed into chemically competent Escherichia coli Transetta (DE3) cells (Transgen). The target proteins GST-HEL, His-ClsHSP, and His-NbsHSP and the control proteins GST and His were expressed after induction by adding $0.5 \mathrm{mmol} /$ liter isopropyl$\beta$-d-thiogalactoside (IPTG) to the medium, and then, they were purified using GST Sefinose Resin and an Ni-NTA-Sefinose Column (Sangon), respectively. Equal amounts of purified protein (e.g., GST and His-NbsHSP, GST-HEL and His-NbsHSP, GST-HEL and His, GST and His-ClsHSP, GST-HEL and His-ClsHSP, and GST and His) were mixed and incubated for $2 \mathrm{~h}$ at $4^{\circ} \mathrm{C}$ in a glutathione sefinose resin prepacked column. The resin was washed three times with $5 \mathrm{ml}$ binding/wash buffer, and the GST-tagged proteins were eluted with $2 \mathrm{ml}$ elution buffer. The eluted proteins were analyzed by western blotting as described previously (Jiang et al. 2014). Briefly, protein samples were separated in $12 \%$ sodium dodecyl sulfate polyacrylamide gel electrophoresis (SDS-PAGE) gels, transferred onto $0.22-\mu \mathrm{m}$ Immobilon-NC Transfer Membranes (Solarbio) by wet electroblotting, and detected with anti-GST or anti-6X His tag antibodies (Abcam) and horseradish peroxidase (HRP)-goat antirabbit immunoglobulin G secondary antibody (EarthOx). The antigen-antibody complexes were flashed using an EasySee Western Blot Kit (Transgen) in accordance with the manufacturer's instructions and imaged using a Chemiluminescence Imaging System (Tanon).

In vivo coimmunoprecipitation assay. For the in vivo coimmunoprecipitation (Co-IP) assay, the fusion fragments FLAGHEL, cMYC-NbsHSP, and cMYC-ClsHSP were obtained after two PCR amplifications with primer pairs CI-HEL-F/CI-HEL-R and CI-FLAG-F/CI-HEL-R, CI-NbsHSP-F/CI-NbsHSP-R and CIcMYC-F/CI-NbsHSP-R, and CI-ClsHSP-F/CI-ClsHSP-R and CIcMYC-F/CI-ClsHSP-R, respectively. Thereafter, fragments were inserted into the NcoI/BstEII sites of a pCambia1302 vector using the In-Fusion HD Cloning Kit to create the recombinant plasmids pCambia1302-FLAG-HEL, pCambia1302-cMYC-NbsHSP, and pCambia1302-cMYC-ClsHSP. These recombinant plasmids were transformed into A. tumefaciens strain GV3101. GV3101 strains carrying pCambia1302-FLAG-HEL, pCambia1302-cMYCNbsHSP, pCambia1302-cMYC-ClsHSP, pCambia1302-FLAG-HEL/ pCambia1302-cMYC-NbsHSP, and pCambia1302-FLAG-HEL/ pCambia1302-cMYC-ClsHSP mixture were infiltrated into $N$. benthamiana leaves. After transient expression for $72 \mathrm{~h}$, the infiltrated leaves were harvested and used for total protein extraction. The in vivo Co-IP assay was performed using a Pierce Immunoprecipitation (Co-IP) Kit following the manufacturer's recommendations (Thermo). Briefly, anti-FLAG and anti-cMYC antibodies (Abcam) were immobilized onto Aminolink Plus coupling resin prepacked in a Pierce spin column. Extracted total protein was added to the appropriate resin and incubated overnight at $4^{\circ} \mathrm{C}$ with gentle mixing. The following day, the resin was washed three times with $200 \mu \mathrm{l}$ of immunoprecipitation (IP) lysis/wash buffer and eluted with $10 \mu \mathrm{l}$ of elution buffer. Eluted samples were analyzed by western blotting using anti-cMYC and anti-FLAG antibodies.

Subcellular localization assay. The plant expression vectors pBWA-mKATE and pBI121-GFP (green fluorescent protein) were stored in our laboratory. The coding sequences of ClsHSP and NbsHSP were amplified using primer pairs Sub-ClsHSP-F/SubClsHSP-R and Sub-NbsHSP-F/ Sub-NbsHSP-R. Thereafter, the fragments were inserted into the SmaI site of a pBWA-mKATE vector using the In-Fusion HD Cloning Kit to create the recombinant plasmids pBWA-ClsHSP-mKATE and pBWANbsHSP-mKATE. The coding sequence of the HEL domain was amplified with the primer pair Sub-HEL-F/Sub-HEL-R and then, inserted into the $\mathrm{XbaI}$ site of a pBI121-GFP vector using the InFusion HD Cloning Kit to create the recombinant plasmid pBI121HEL-GFP. The aforementioned recombinant plasmids were transformed into A. tumefaciens strain GV3101, and GV3101 strains carrying
pBWA-ClsHSP-mKATE or pBWA-NbsHSP-mKATE were infiltrated into healthy and CGMMV-infected $N$. benthamiana leaves. pBI121-HEL-GFP and the mixture containing pBWA-ClsHSPmKATE/pBI121-HEL-GFP or pBWA-NbsHSP-mKATE/pBI121HEL-GFP were infiltrated into healthy $N$. benthamiana leaves. After infiltration for $72 \mathrm{~h}$, the fluorescence patterns in infiltrated leaves were analyzed using a Leica confocal laser-scanning microscope (Leica). The excitation wavelengths used for mKATE and GFP were 543 and $488 \mathrm{~nm}$, respectively, and the emitted light from mKATE and GFP was captured between 520 and $580 \mathrm{~nm}$, respectively, and 500 and $550 \mathrm{~nm}$, respectively.

Stable plant transformation. To construct an NbsHSP overexpressing vector, the coding sequence of NbsHSP was amplified with the primer pair Overex-NbsHSP-F/Overex-NbsHSP-R using the plasmid AD-NbsHSP as a template, and the amplified fragment was then cloned into a pCambia1300 vector to generate pCambia-Overex-NbsHSP. An NbsHSP hairpin structure consisting of two reversed partial sequences of NbsHSP (corresponding to the amino acid sequence from residue 38 to 110) and a noncoding intron were cloned into the pCambia1300 vector to construct an NbsHSP silencing vector (pCambia-RNAi-NbsHSP). All constructs were introduced into A. tumefaciens strain EHA105. The genus Agrobacterium-mediated transformation of $N$. benthamiana was performed by following a previously published protocol (Horsch et al. 1985). Transgenic $N$. benthamiana seedlings were initially selected on Murashige and Skoog medium supplemented with $50 \mu \mathrm{g} / \mathrm{ml}$ kanamycin. Two weeks thereafter, kanamycin-resistant seedlings were transferred to $10-\mathrm{cm}$ pots filled with a mixture of meadow soil and vermiculite and grown to maturity in a greenhouse. $T_{1}$ plants containing overexpressed or silenced $N b s H S P$, as confirmed by quantitative reverse transcription PCR (qRT-PCR), were used for CGMMV inoculations.

RNA analysis. Total RNA was extracted from $N$. benthamiana and $C$. lanatus leaves using RNAiso Plus (Takara) according to the manufacturer's instructions. Reverse transcription PCR (RT-PCR) analyses for confirmation of CGMMV systemic infection were performed after the synthesis of first-strand cDNA using HEL-R in accordance with the protocol of a Fastking RT Kit (Tiangen). PCR was performed using the primer pair HEL-F/HEL-R to amplify a 765-bp fragment.

For northern blot analyses, the DNA probe used for detecting CGMMV RNA was synthesized as described previously (Liu et al. 2017). The procedures were performed using a DIG Northern Starter Kit according to the manufacturer's instructions (Roche). Hybridization was assessed using the Chemiluminescence Imaging System (Tanon).

The expression of sHSPs was detected by real-time qRT-PCR using the primer pairs RT-ClsHSP40-F/RT-ClsHSP40-R, RTClsHSP65-F/RT-ClsHSP65-R， RT-ClsHSP12-F/RT-ClsHSP12-R, RT-ClsHSP97-F/RT-ClsHSP97-R, RT-ClsHSP45-F/RT-ClsHSP45-R, and RT-NbsHSP-F/RT-NbsHSP-R. The internal reference primers ClCAC-F/ClCAC-R (C. lanatus Actin) and NbUBC-F/NbUBC-R ( $N$. benthamiana Ubiquitin Conjugating Enzyme) were designed based on previous studies (Kong et al. 2014; Schmidt and Delaney 2010) (Supplementary Table S1). The qRT-PCRs were performed using a Roche LightCycler 480II Real-Time PCR system (Roche), and the amplified products were analyzed using the $\Delta \Delta C_{\mathrm{T}}$ method.

\section{RESULTS}

The CGMMV HEL domain interacts with a host sHSP in yeast. To identify the host factor(s) interacting with the CGMMV HEL domain during viral infection, a $C$. lanatus leaf-specific cDNA library was constructed from watermelon seedlings infected with CGMMV. The library titer and recombination rate were $5.4 \times 10^{8}$ $\mathrm{CFU} / \mathrm{ml}$ and $100 \%$, respectively. The average length of the inserted fragments was $\sim 1 \mathrm{kbp}$. These results indicated that we had constructed a high-quality library that could be used for screening. 
We then inserted the CGMMV HEL coding region into the vector pGBKT7 (pGBKT-HEL) for expression as a GAL4 fusion and used this as the bait to screen the watermelon cDNA library. We accordingly succeeded in identifying a single positive clone. The nucleotide sequence of this clone was blasted in the cucurbit genomic database and showed $100 \%$ homology with a watermelon gene (Cla017945) that presumably encodes an sHSP, which we designated ClsHSP (GenBank accession number HQ681888). We cloned the coding region of ClsHSP and that of the closest homolog of this sequence in $N$. benthamiana (designated NbsHSP; XM_016604898.1). Using a yeast two-hybrid assay, we demonstrated that NbsHSP interacts with the viral HEL domain, and we similarly confirmed positive interaction between the fulllength ClsHSP and the HEL domain (Fig. 1A). Because the HEL domain is one of the domains of the $129-\mathrm{kDa}$ protein, we further examined the interaction between the sHSP and the 129-kDa protein, MT domain, or IR region. The results showed that, whereas sHSP interacts with the HEL domain and the 129-kDa protein, no interaction could be detected between this sHSP and either the MT domain or the
IR region, which indicates the specificity of the interaction between the sHSP and the HEL domain during CGMMV infection (Fig. 1B).

GST pull-down and Co-IP analyses of the interaction between the CGMMV HEL domain and sHSP. The interaction between the CGMMV HEL domain and the sHSP was further confirmed based on an in vitro GST pull-down assay. The GST-tagged HEL (GST-HEL) domain and the His-tagged sHSP (His-ClsHSP or His-NbsHSP) were expressed in Transetta (DE3) cells. Equal amounts of purified GST-HEL and His-sHSP were mixed and then pulled down with GST binding beads followed by western blotting using anti-GST or anti-His antibodies. In these experiments, the combinations of GST/His-ClsHSP, GST/HisNbsHSP, GST-HEL/His, and GST/His were used as controls. The results of these experiments showed that only GST-HEL could pull down His-sHSP (Fig. 2A), thereby demonstrating the in vitro interaction between the CGMMV HEL domain and the sHSP.

Subsequently, we used a Co-IP assay to examine the in vivo interaction between the two proteins. Constructs designed to express FLAG-HEL, cMYC-ClsHSP, cMYC-NbsHSP, FLAG-HEL/

A

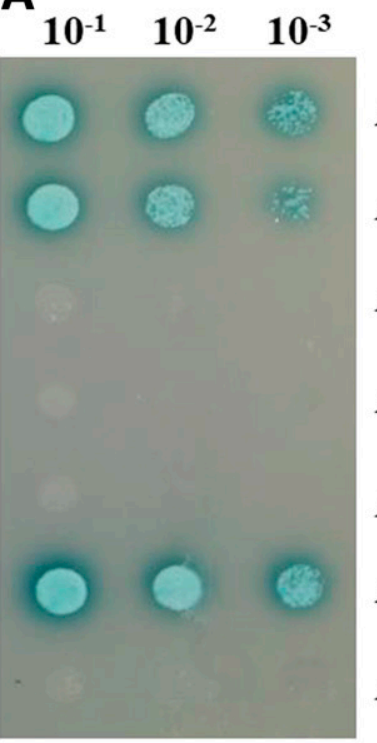

AD-CIsHSP+BD-HEL

AD-NbsHSP+BD-HEL

AD-ClsHSP+BD

AD-NbsHSP+BD

AD+BD-HEL

AD-T+BD-53

AD-T+BD-Lam

B

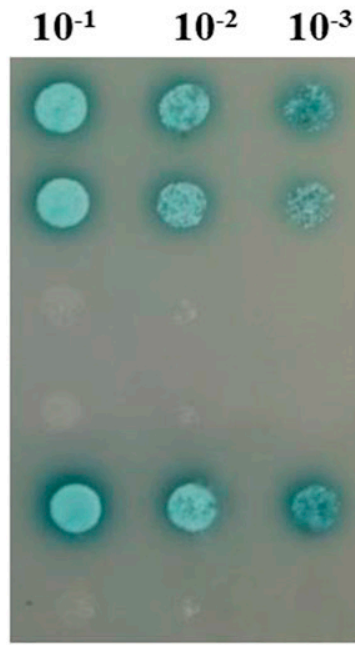

AD-CIsHSP+BD-HEL

AD-CIsHSP+BD-129

$\begin{array}{lll}10^{-1} & 10^{-2} & 10^{-3}\end{array}$

AD-CIsHSP+BD-MT

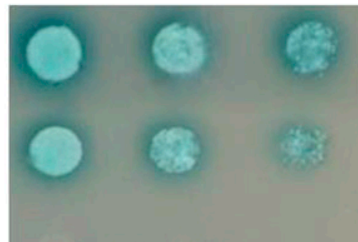

AD-NbsHSP+BD-HEL

AD-NbsHSP+BD-129

AD-NbsHSP+BD-MT

AD-ClsHSP+BD-IR

AD-NbsHSP+BD-IR

AD-T+BD-53

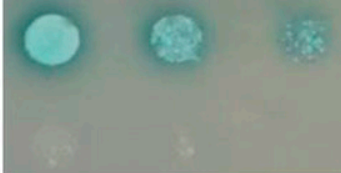

AD-T+BD-53

\section{AD-T+BD-Lam}

\section{AD-T+BD-Lam}

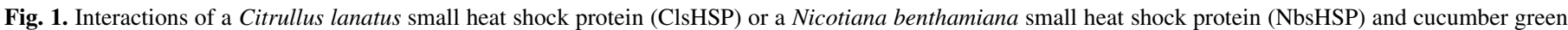

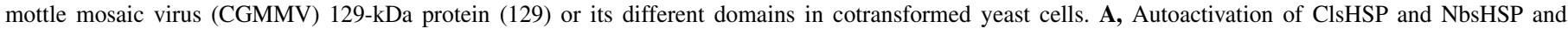

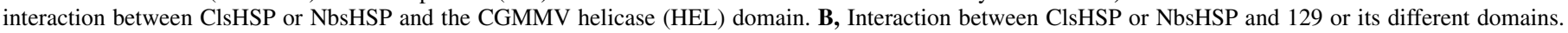

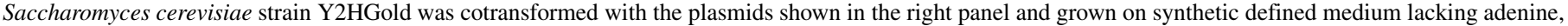
histidine, leucine, and tryptophan with $40 \mu \mathrm{g} / \mathrm{ml} \mathrm{X- \alpha -Gal} \mathrm{(SD/-Ade/-His/-Leu/-Trp/X-} \alpha$-Gal) at three different concentrations. 
cMYC-ClsHSP, and FLAG-HEL/cMYC-NbsHSP were the genus Agrobacterium infiltrated into $N$. benthamiana leaves, and total proteins were extracted from $N$. benthamiana leaves and subsequently used in Co-IP assays at $72 \mathrm{~h}$ postinfiltration. As shown in Figure 2B, FLAG-HEL coimmunoprecipitated with either cMYCClsHSP or cMYC-NbsHSP. The interactions were confirmed by performing the reciprocal experiment, which duly indicated that cMYC-ClsHSP or cMYC-NbsHSP could coimmunoprecipitate with FLAG-HEL. These results, therefore, provide convincing evidence that CGMMV HEL also interacts with ClsHSP or NbsHSP in vivo, further supporting a biologically relevant interaction between the virus and plant proteins.

sHSPs are induced in the area of initial CGMMV infection. The $C$. lanatus genome contains 20 putative genes that harbor an sHSP domain, 11 of which are class I sHSPs. We used RTPCR to analyze the expression of these 11 genes in healthy and CGMMV-infected plants, and we reliably detected transcripts for five of the 11 class I sHSPs, whereas the other six were undetectable in either CGMMV- or mock-infected watermelon plants, which can probably be attributed to their specific induction under different conditions and/or in other tissues. To confirm whether CGMMV infection can induce the expression of host sHSPs, we used qRT-PCR to examine changes in the expression patterns of these five endogenous sHSPs in CGMMV-inoculated $C$. lanatus leaves at 2 days postinoculation (dpi). The results indicated that three sHSPs were upregulated, whereas the remaining two exhibited no obvious change in expression. Among the three sHSPs with increased expression, ClsHSP (Cla017945) showed the highest expression, with a sixfold change compared with the mock-inoculated plants. However, at $14 \mathrm{dpi}$, when CGMMV symptoms initially appeared on systemically infected $C$. lanatus plants, the three sHSPs exhibited no obvious change in expression (Fig. 3A). We also examined the expression level of NbsHSP in CGMMV-inoculated $N$. benthamiana leaves at 2 dpi and systemically leaves at $7 \mathrm{dpi}$, when CGMMV symptoms initially appeared on the latter leaves, and we found that NbsHSP expression was highly consistent with that of ClsHSP (Fig. $3 \mathrm{~B})$. These results accordingly indicate that SHSPs are transcriptionally induced in the area of initial CGMMV infection but not in systemically infected leaves.

The CGMMV HEL domain affects sHSP subcellular localization through interaction. To assay the subcellular localization of ClsHSP and NbsHSP, ClsHSP or NbsHSP fused to mKATE at their C termini was transiently expressed in healthy or CGMMV-infected $N$. benthamiana epidermal cells. Observation of mKATE fluorescence revealed that both proteins formed numerous granules in the cytoplasm of the healthy cells, whereas we were unable to detect these granules in CGMMV-infected cells (Fig. 4A). We also inserted the cDNA sequence of the HEL domain into the pBI121-GFP vector, and after transient expression in N. benthamiana epidermal cells, we observed that GFP fluorescence was localized in
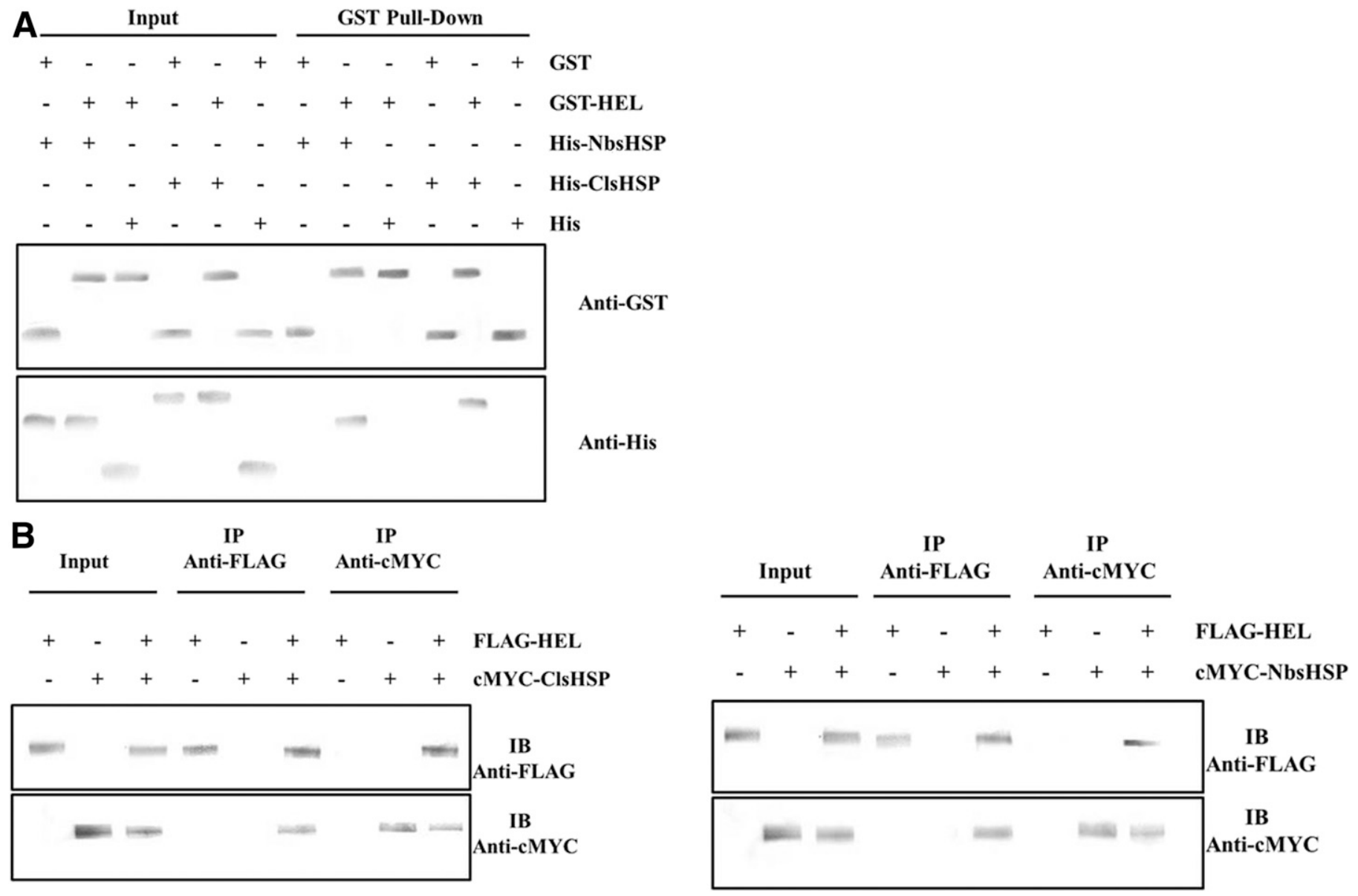

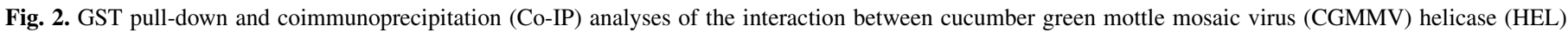

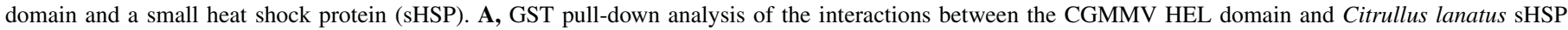

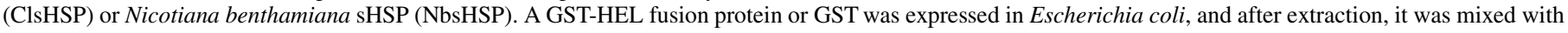

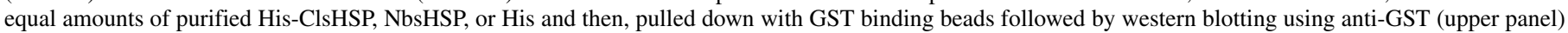

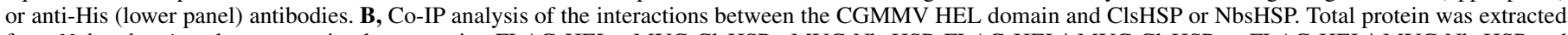

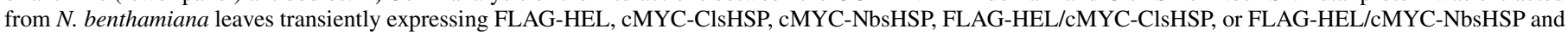

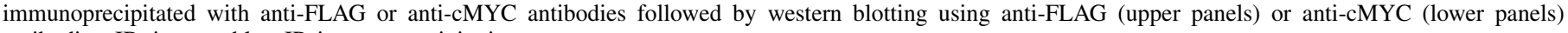
antibodies. IB, immunoblot; IP, immunoprecipitation. 
both the nucleus and cytoplasm (Fig. 4B). To further establish whether the change in sHSP distribution is related to an interaction with the HEL domain, the latter was coexpressed with ClsHSP or NbsHSP in $N$. benthamiana epidermal cells, and we found that the distributions of ClsHSP and NbsHSP were consistent with their distribution in the CGMMV-infected $N$. benthamiana epidermal cells (Fig. 4C). Whereas the HEL domain and sHSP were colocalized in the cytoplasm, coexpression had no influence on the nuclear localization of the HEL domain, suggesting that the interaction occurs specifically in the cytoplasm. These results accordingly indicate that, by interacting with a host sHSP, the CGMMV HEL domain can affect the subcellular localization of this sHSP.

Alteration of $\mathrm{Nbs} H S P$ expression affects CGMMV symptom development and RNA accumulation. $N$. benthamiana is an excellent experimental host for CGMMV, providing a simple genetic transformation system for studying the function of proteins interacting with CGMMV. To confirm the function of sHSPs during CGMMV infection, we generated NbsHSP-silenced transgenic $N$. benthamiana (RNAi-NbsHSP) plants and NbsHSP overexpressing transgenic $N$. benthamiana (35S-NbsHSP) plants. Under the growth chamber conditions used in this study, RNAi-NbsHSP and 35S-NbsHSP transgenic $N$. benthamiana plants showed normal growth comparable with that of WT plants. The results of qRT-PCR indicated that the expression level of NbsHSP in RNAi-NbsHSP $N$. benthamiana plants was significantly suppressed, whereas it increased significantly in 35S-NbsHSP $N$. benthamiana plants relative to that in the WT plants (Fig. 5A). For the purposes of subsequent research, we selected a single line for which the sHSP silencing efficiency was highest among the 12 RNAi-NbsHSP lines and one line for which sHSP expression was highest among the 15 35S-NbsHSP lines.

To analyze the role of the sHSP during CGMMV infection, we inoculated WT, RNAi-NbsHSP, and 35S-NbsHSP transgenic $N$. benthamiana plants via mechanical inoculation or the genus Agrobacterium infiltration of an infectious clone. We found that symptoms of CGMMV appeared on systemically infected leaves of RNAi-NbsHSP transgenic and WT N. benthamiana plants at 6 dpi.
Moreover, we detected similar levels of viral RNA accumulation in these plants. In contrast, 35S-NbsHSP transgenic plants were symptomless until $11 \mathrm{dpi}$, at which time the symptoms in RNAiNbsHSP transgenic and WT plants were already severe (Fig. 5B). The results of a northern blot assay were consistent with the development of disease symptoms. At $11 \mathrm{dpi}$, when the $35 \mathrm{~S}-$ NbsHSP transgenic plants showed the initial symptoms of disease, CGMMV RNAs were detected in the 35S-NbsHSP transgenic plants, although their accumulation was lower than that in WT and RNAi-NbsHSP transgenic plants (Fig. 5C). These results were reproducible using either mechanical inoculation or Agrobacteriummediated inoculation, implying either that any effects of Agrobacterium tumefaciens did not affect the findings or that no such effect occurred. Collectively, our results clearly indicate that sHSP overexpression affects the accumulation of CGMMV RNA and the subsequent development of disease symptom in infected plants.

\section{DISCUSSION}

During pathogens invasion, plants utilize a wide range of responses as protective measures against infection. Expression of HSPs is one of the effective responses that enable plant cells to adapt to environmental changes and survive adverse conditions (Gupta et al. 2010). To establish successful infection, pathogens use a series of countermeasures to suppress host defenses. Characteristically, bacteria and fungi introduce effector molecules into plant cells to modulate cellular functions and thereby, disrupt host defense responses (Ahmed et al. 2016). However, viruses, which are obligate parasites, will also adopt a variety of strategies to promote their multiplication and dispersal. In this study, using yeast twohybrid assays, we detected a host sHSP that interacts with the HEL domain of CGMMV. This interaction was further verified both in vitro and in vivo. On the basis of these observations, we subsequently performed a number of experiments to elucidate the role of this sHSP during CGMMV infection. We accordingly demonstrated that changes in the distribution pattern of the sHSP were closely associated with expression of the viral HEL domain,
A

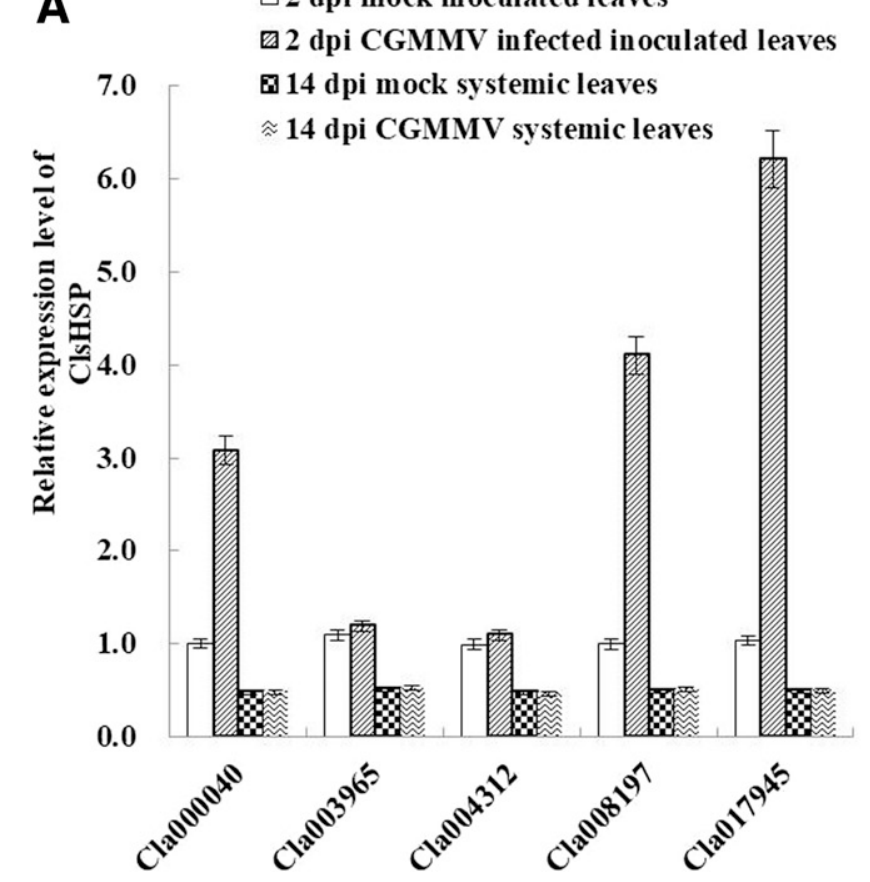

$\square 2$ dpi mock inoculated leaves Q 2 dpi CGMMV infected inoculated leaves
B
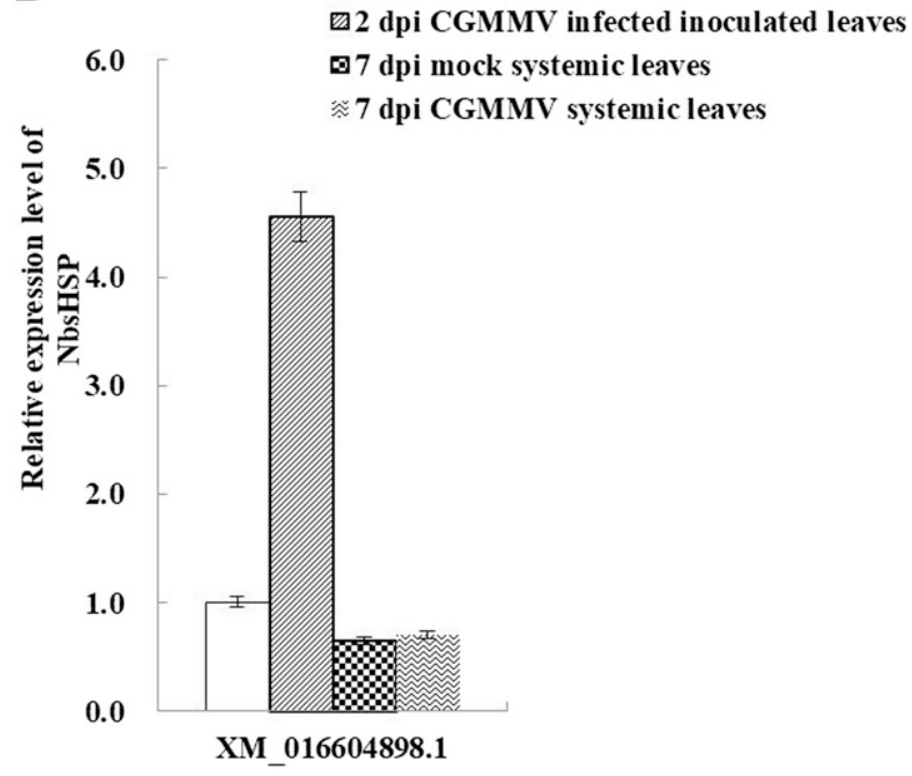

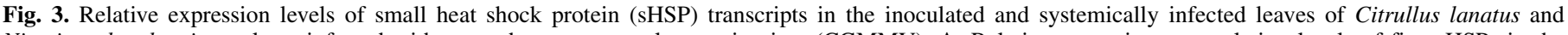

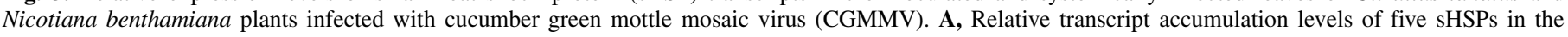

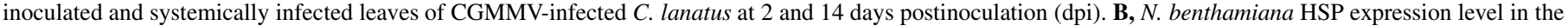
inoculated and systemically infected leaves of CGMMV-infected $N$. benthamiana at 2 and 7 dpi. Experiments were repeated three times. 
which indicates that, as a new countermeasure against host defense response, CGMMV alters host protein biological functions by disturbing their subcellular distribution. To the best of our knowledge, there have been no previous descriptions of this type of host modulation with respect to tobamovirus infection.

HSPs occur ubiquitously in plant and animal cells and are essential components involved in the control of cellular homeostasis under both optimal and adverse growth conditions. They function in protein folding, assembly, translocation, and degradation during normal cellular growth and development, and they are also responsible for protein stabilization and refolding in cells under stress (Lindquist and Craig 1988; Wang et al. 2004; Whitley et al. 1999). sHSPs are generally considered to be stress-related defense proteins that respond to a range of abiotic or biotic stress conditions (Efeoğlu
2009). They are known to stabilize several intracellular proteins, including defense-related signaling components, through their chaperone activity (Ahmed et al. 2015). sHSPs are also referred to as "holdases," the characteristic features of which include acting as ATP-independent molecular chaperones that bind to denatured proteins or newly synthesized proteins to prevent them from forming irreversible aggregations under stress conditions (Zhang et al. 2015).

Although under normal growth conditions, sHSPs are generally undetectable in vegetative tissues, they can be significantly induced by environmental stresses or developmental stimuli (Sun et al. 2002). Previously, it has been shown that, in Oryza sativa and A. thaliana, host sHSP transcript levels increase after infection by different viruses, indicating that the induction of host sHSP expression
A
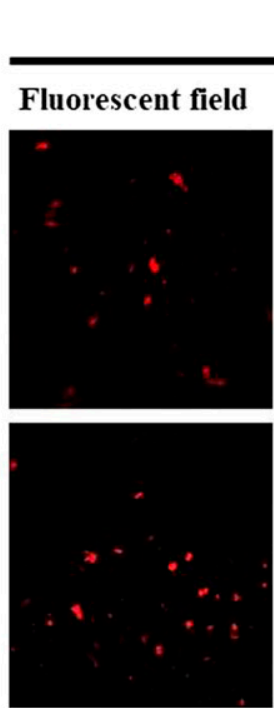

B

HEL-GFP

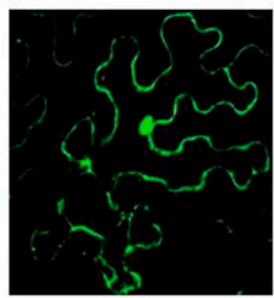

Fluorescent field

C

CIsHSPmKATE/ HEL-GFP

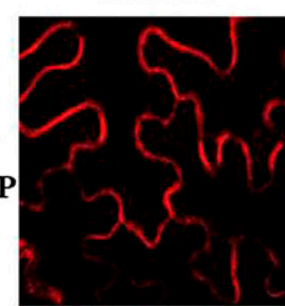

NbsHSPmKATE/ HEL-GFP

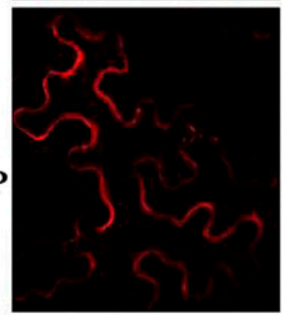

Mock
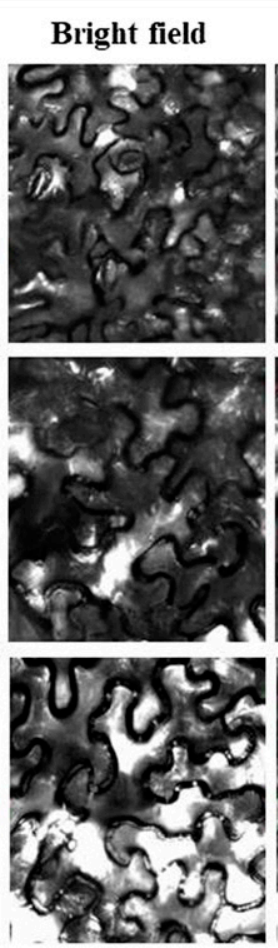

Fluorescent field
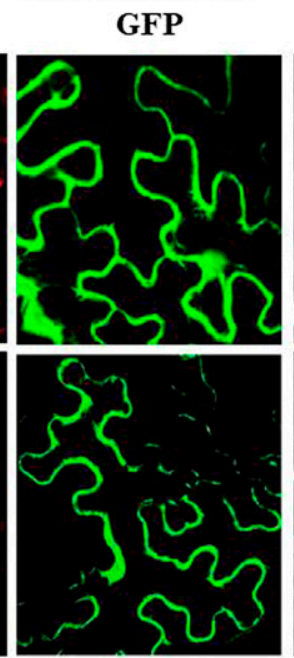

Bright field
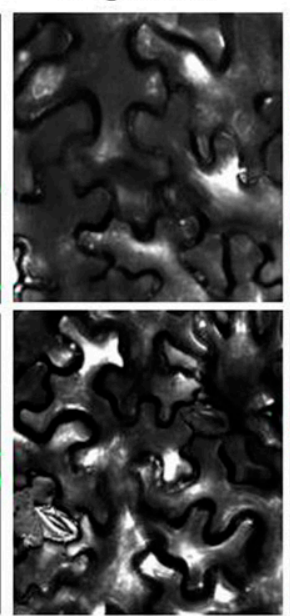

\section{CGMMV-infected}
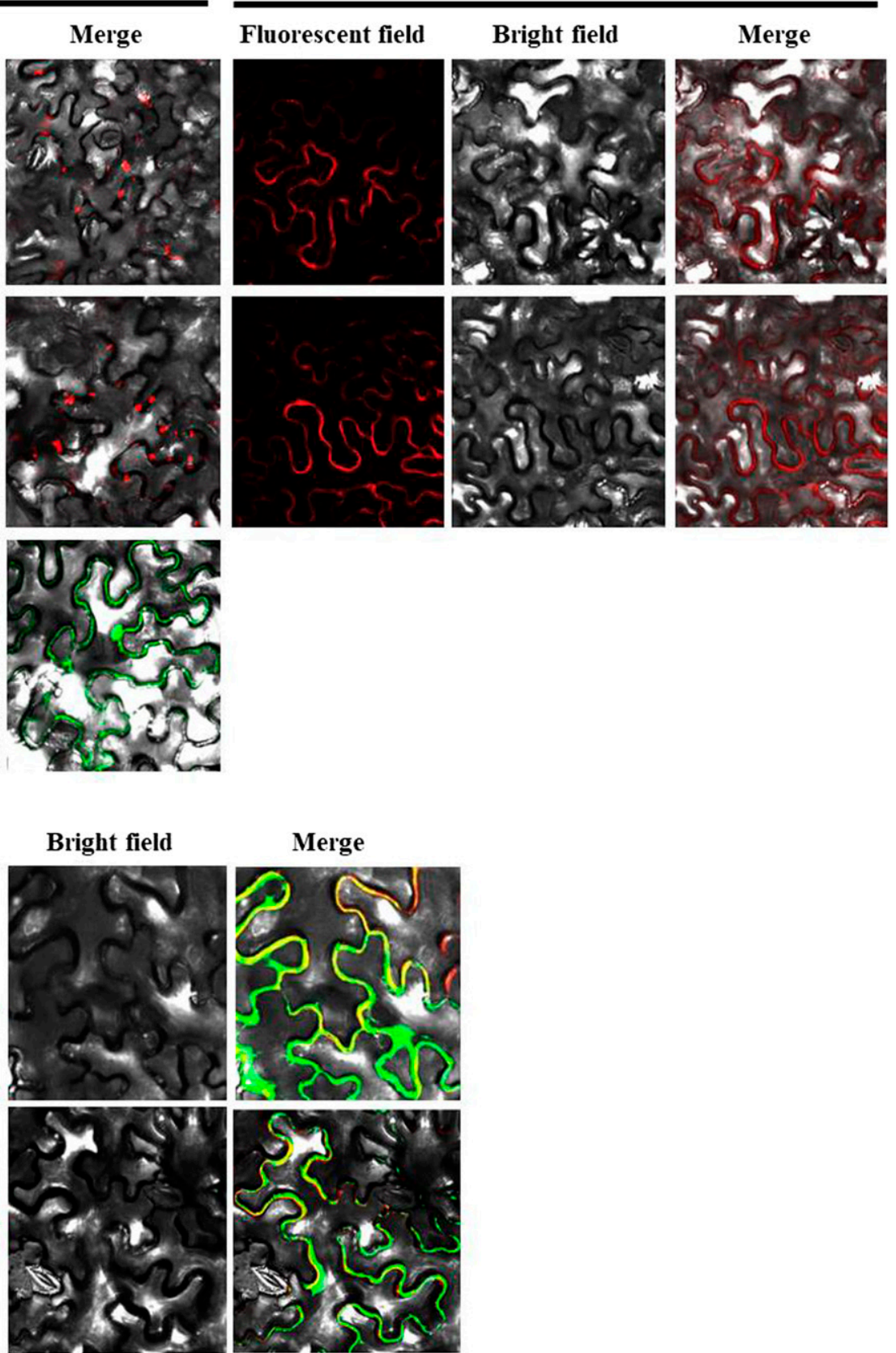

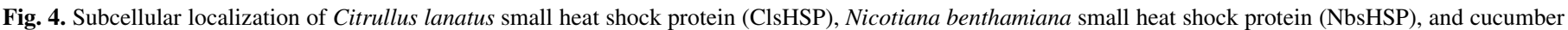

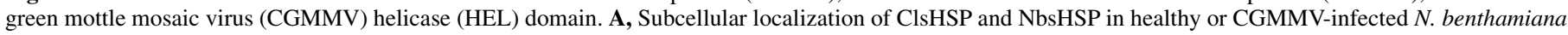

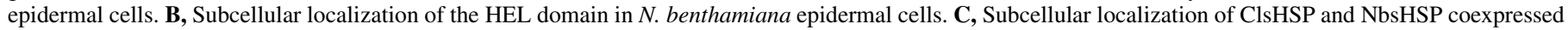
with the HEL domain. 
might be a common response during viral infection (Li et al. 2015; Whitham et al. 2003). sHSPs also play a fundamental role in protecting animal cells during viral infection (Piotrowska et al. 2010). In this study, we found that host plant sHSPs are upregulated in the area of initial CGMMV infection, which is consistent with the findings of previous studies that they have demonstrated the upregulation of these proteins in response to viral infection in $O$. sativa and $A$. thaliana. Nevertheless, we found that, in systemically infected leaves, sHSP expression returns to normal levels concomitant with development of the initial disease symptoms, which indicates that sHSPs may play roles associated with the host basal defense response to CGMMV infection.

Previously, there have been a few studies that have examined the interaction of sHSPs with plant viruses. An sHSP interacts with the RSV Pc4 protein, and this interaction may contribute to maintaining the denatured $\mathrm{Pc} 4$ competent for transport through plasmodesmata. Moreover, the presence of the sHSP would facilitate the renaturation of viral material on entering an adjacent cell ( $\mathrm{Lu}$ et al. 2009). The interaction between a host sHSP and the RSV RdRp has also been found to alter the subcellular localization of the sHSP, and it suppresses the formation of stress-related granules in the cytoplasm, which may represent a mechanism whereby the virus disrupts host antiviral responses ( $\mathrm{Li}$ et al. 2015). Consistent with the findings of $\mathrm{Li}$ et al. (2015), a subcellular localization assay performed in this study revealed that sHSPs formed granules in healthy cells and that the distribution was altered in response to expression of the HEL domain within these cells. In response to stress, sHSPs form granules by interacting with their protected proteins and mRNAs to maintain plant fitness. It can accordingly be hypothesized that, during CGMMV infection, inhibition of granule formation may be an effective mechanism whereby the virus antagonizes host defense responses; however, whether the host proteins protected by the sHSP are exploited for the benefit of viral infection must await further clarification.

We also demonstrated that sHSP silencing in NbsHSP transgenic $N$. benthamiana plants had no significant effect on CGMMV infection, with transgenic plants showing symptoms and viral RNA accumulation similar to those observed in WT plants. It is conceivable that sHSP functional redundancy may underlie this phenotype, because there are numerous sHSP homologs that may perform similar functions. However, compared with WT plants inoculated with CGMMV, those plants overexpressing NbsHSP showed delayed systemic symptoms, and northern blot results revealed that CGMMV RNA accumulation was negatively correlated with sHSP expression. Thus, upregulation of plant sHSP expression may be an effective plant defense strategy deployed in response to CGMMV infection.

Additional roles of sHSPs in host defense responses have been suggested based on the findings of studies showing that sHSPs can activate autophagy in mammalian cells (Liang et al. 2018), which is known to contribute to innate immune responses through the elimination of intracellular pathogens, including viruses. Previous
A

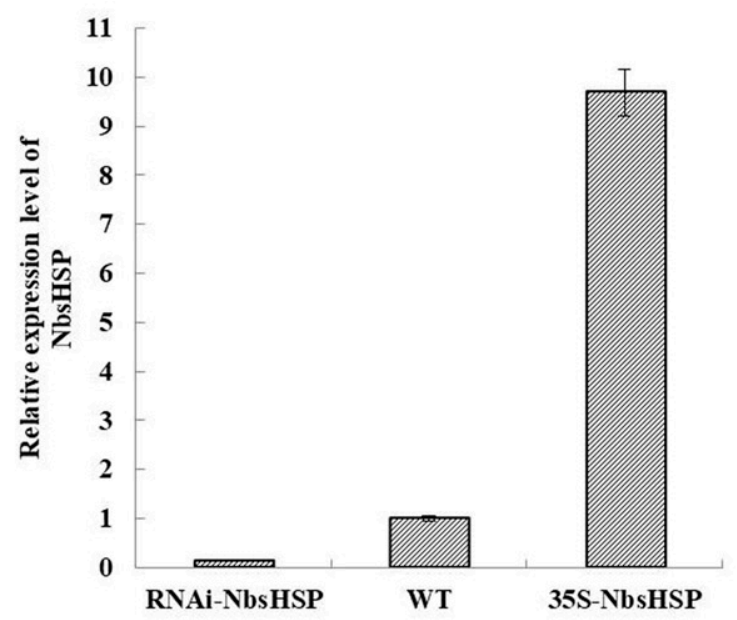

B Mechanical inoculation

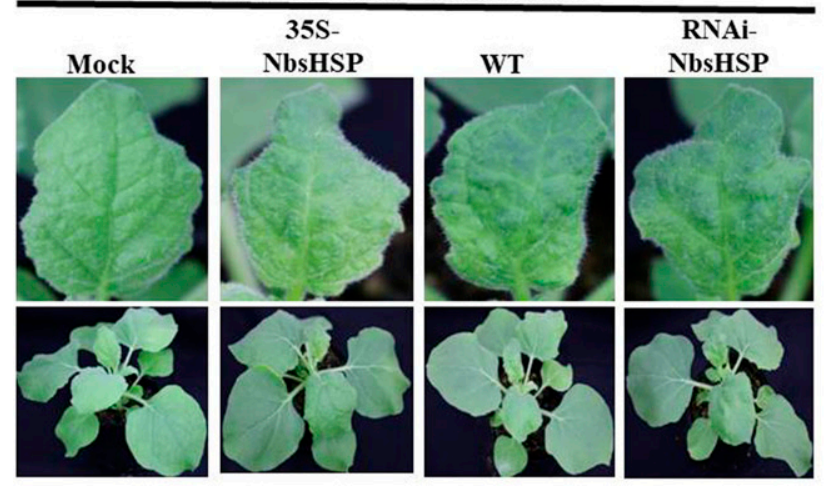

C $\frac{\text { Mechanical inoculation }}{\text { RNAi- }} \frac{\text { Infectious clone }}{35 \mathrm{~S}-}$ Mock NbsHSP WT NbsHSP Mock NbsHSP WT NbsHSP

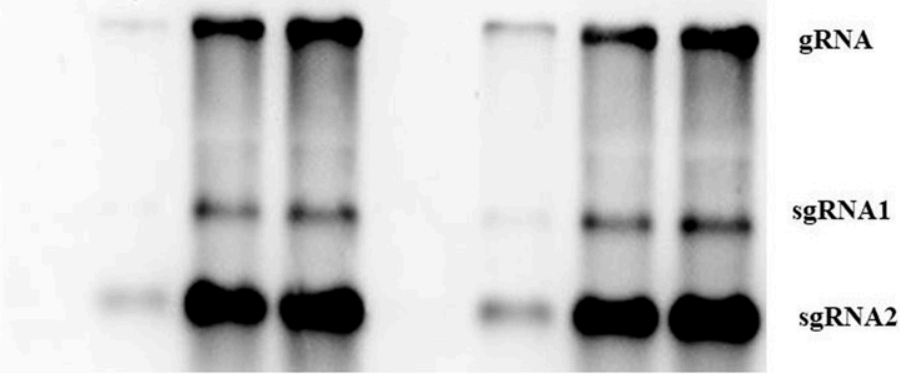

gRNA

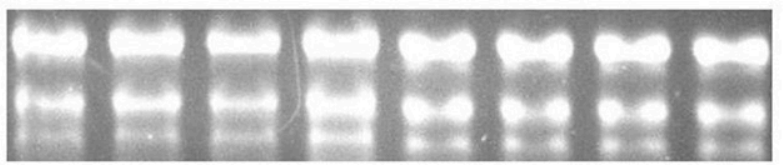

rRNA

Infectious clone

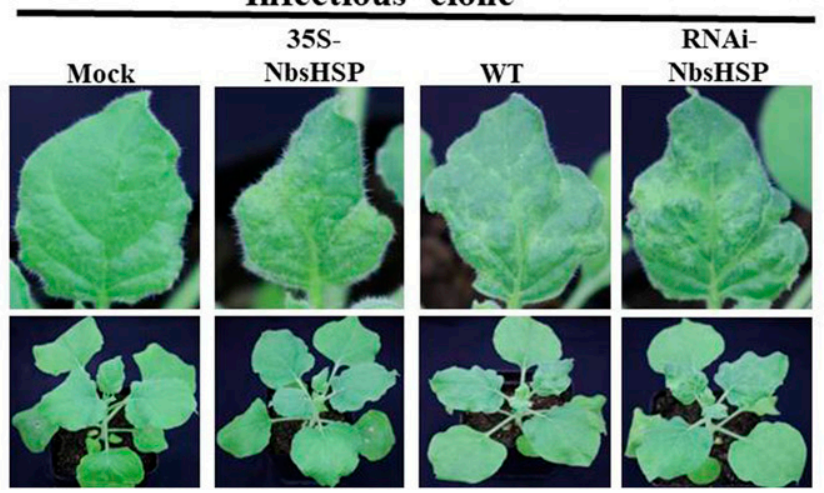

Fig. 5. Cucumber green mottle mosaic virus (CGMMV) symptom development and RNA accumulation in transgenic and wild-type (WT) Nicotiana benthamiana plants. A, Relative expression levels of $N$. benthamiana small heat shock protein (NbsHSP) transcripts in RNAi-NbsHSP, WT, and 35S-NbsHSP N. benthamiana plants. B, Symptoms in systemically infected leaves of 35S-NbsHSP, WT, and RNAi-NbsHSP $N$. benthamiana plants inoculated with CGMMV at 11 days postinoculation (dpi). C, Northern blot analysis of CGMMV RNA accumulation in the systemically infected leaves of 35S-NbsHSP, WT, and RNAi-NbsHSP $N$. benthamiana plants inoculated with CGMMV at $11 \mathrm{dpi}$ 
studies have shown that autophagic mechanisms are antagonized or even exploited by a number of animal viruses (Dong and Levine 2013), and autophagy has begun to be studied with respect to plant-virus interactions (Cheng and Wand 2016; Hafrén et al. 2017, 2018). sHSPs can induce the catalytic activity of the $26 \mathrm{~S}$ proteasome, which degrades ubiquitinated proteins under stress conditions (Lanneau et al. 2010), and in yeast, the 26S proteasome has been found to be degraded by autophagy-related protein 8-mediated autophagy (Marshall et al. 2016). Accordingly, the possibility that the sHSP interactions identified for CGMMV in this study are indirectly related to host autophagy via mediation of $26 \mathrm{~S}$ proteasome catalytic activity is an attractive hypothesis worthy of additional investigation. Thus, although the interaction between the host sHSP and the viral protein has been convincingly established, the specific biological function of the sHSP during CGMMV infection needs to be further examined.

RNA virus HEL domains are generally considered to be motor proteins involved in the unwinding of double-stranded RNA (dsRNA) replication intermediates and destabilization of strong secondary structures via NTP hydrolysis during virus replication (Caruthers and Mckay 2002). The CGMMV HEL domain is one of the domains present in both 129- and 186-kDa replicases that are generated via direct translation of viral genomic RNAs (gRNAs) during early infection. As a critical regulator of viral infection, the HEL domain in tobamovirus is implicated in the regulation of several processes via its interaction with host factors during viral infection, including viral multiplication, symptom development, host defense responses, and $N$ gene resistance (Padmanabhan et al. 2005; Ueda et al. 2006; Wang et al. 2009; Yamanaka et al. 2002). The interaction between the CGMMV HEL domain and host plant sHSP may be related to the disruption of host plant basal defense responses to facilitate viral infection. The multifunctional nature of the HEL domain suggests that it participates in several host biological processes to accelerate viral infection. Given the functional versatility of the HEL domain in the viral lifecycle, it has been proposed as an ideal single-hit target to inhibit coinfection of the human immunodeficiency virus and hepatitis $\mathrm{C}$ virus (Anwar et al. 2018). Similarly, the HEL domain may be an important target for future plant antiviral strategies.

Although host factors are acknowledged to be key components in viral infection, there have been no reports of host factors that interact with CGMMV proteins. In this study, we identified a host sHSP that interacts with the CGMMV HEL domain. This interaction was closely associated with the subcellular relocalization of the sHSP, the accumulation of CGMMV RNA, and the development of disease symptoms. We suspect that the identified sHSP may form part of a defense line that is deployed against CGMMV during the initial stages of viral infection. Thus, our study may provide a new theoretical basis for the breeding of CGMMV-resistant plants.

\section{LITERATURE CITED}

Abdelhaleem, M. 2009. Helicases: An overview. Pages 1-12 in: Helicases. Springer.

Ahmed, A. A., Mclellan, H., Aguilar, G. B., Hein, I., Thordal-Christensen, H., and Birch, P. R. 2016. Engineering barriers to infection by undermining pathogen effector function or by gaining effector recognition. Pages 23-50 in: Plant Pathogen Resistance Biotechnology.D. B.Collinge, ed. Wiley, Hoboken, NJ.

Ahmed, A. A., Pedersen, C., Schultz-Larsen, T., Kwaaitaal, M., Jørgensen, H. J., and Thordal-Christensen, H. 2015. The barley powdery mildew candidate secreted effector protein CSEP0105 inhibits the chaperone activity of a small heat shock protein. Plant Physiol. 168:321-333.

Anwar, M. F., Zarina, S., Ali, S., and Abidi, S. H. 2018. Two for one: Viral helicases as an ideal target for HIV and HCV co-infection. Med. Hypotheses 116:139-140.

Asano, M., Satoh, R., Mochizuki, A., Tsuda, S., Yamanaka, T., Nishiguchi, M., Hirai, K., Meshi, T., Naito, S., and Ishikaws, M. 2005. Tobamovirusresistant tobacco generated by RNA interference directed against host genes. FEBS Lett. 579:4479-4484.
Basha, E., Jones, C., Wysocki, V., and Vierling, E. 2010. Mechanistic differences between two conserved classes of small heat shock proteins found in the plant cytosol. J. Biol. Chem. 285:11489-11497.

Beier, H., Barciszewska, M., Krupp, G., Mitnacht, R., and Gross, H. J. 1984. UAG readthrough during TMV RNA translation: Isolation and sequence of two tRNAsTyr with suppressor activity from tobacco plants. EMBO J. 3: 351-356.

Bilgin, D. D., Liu, Y., Schiff, M., and Dineshkumar, S. P. 2003. P58 ${ }^{\mathrm{IPK}}$, a plant ortholog of double-stranded RNA-dependent protein kinase PKR inhibitor, functions in viral pathogenesis. Dev. Cell 4:651-661.

Caruthers, J. M., and Mckay, D. B. 2002. Helicase structure and mechanism. Curr. Opin. Struct. Biol. 12:123-133.

Cheng, X., and Wand, A. 2016. The potyvirus silencing suppressor protein VPg mediates degradation of SGS3 via ubiquitination and autophagy pathways. J. Virol. 91:e01478-16.

Dombrovsky, A., Tran-Nguyen, L. T., and Jones, R. A. 2017. Cucumber green mottle mosaic virus: Rapidly increasing global distribution, etiology, epidemiology, and management. Annu. Rev. Phytopathol. 55:231-256.

Dong, X., and Levine, B. 2013. Autophagy and viruses: Adversaries or allies? J. Innate Immun. 5:480-493.

Efeoğlu, B. 2009. Heat shock proteins and heat shock response in plants. Gazi Univ. J. Sci. 22:67-75.

Escobar, C., Barcala, M., Portillo, M., Almoguera, C., Jordano, J., and Fenoll, C. 2003. Induction of the Hahsp17.7G4 promoter by root-knot nematodes: Involvement of heat-shock elements in promoter activity in giant cells. Mol. Plant-Microbe In. 16:1062-1068.

Fujisaki, K., Ravelo, G. B., Naito, S., and Ishikawa, M. 2006. Involvement of THH1, an Arabidopsis thaliana homologue of the TOM1 gene, in tobamovirus multiplication. J. Gen. Virol. 87:2397-2401.

García, J. A., and Pallás, V. 2015. Viral factors involved in plant pathogenesis. Curr. Opin. Virol. 11:21-30.

Gietz, R. D., and Woods, R. A. 2002. Transformation of yeast by lithium acetate/single-stranded carrier DNA/polyethylene glycol method. Methods Enzymol. 350:87-96.

Goodman, A. G., Fornek, J. L., Medigeshi, G. R., Perrone, L. A., Peng, X., Dyer, M. D., Proll, S. C., Knoblaugh, S. E., Carter, V. S., and Korthe, M. J. 2009. P58IPK: A novel "CIHD" member of the host innate defense response against pathogenic virus infection. PLoS Pathog 5:e1000438.

Gorbalenya, A. E., and Koonin, E. V. 1993. Helicases: Amino acid sequence comparisons and structure-function relationships. Curr. Opin. Struct. Biol. 3:419-429.

Goregaoker, S. P., and Culver, J. N. 2003. Oligomerization and activity of the helicase domain of the tobacco mosaic virus 126- and 183-kilodalton replicase proteins. J. Virol. 77:3549-3556.

Gupta, S. C., Sharma, A., Mishra, M., Mishra, R. K., and Chowdhuri, D. K. 2010. Heat shock proteins in toxicology: How close and how far? Life Sci. $86: 377-384$

Hafrén, A., Macia, J. L., Love, A. J., Milner, J. J., Drucker, M., and Hofius, D. 2017. Selective autophagy limits cauliflower mosaic virus infection by NBR1-mediated targeting of viral capsid protein and particles. Proc. Natl. Acad. Sci. USA 114:E2026-E2035.

Hafrén, A., Üstün, S., Hochmuth, A., Svenning, S., Johansen, T., and Hofius, D. 2018. Turnip mosaic virus counteracts selective autophagy of the viral silencing suppressor HCpro. Plant Physiol. 176:649-662.

Hartl, F. U., Bracher, A., and Hayer-Hartl, M. 2011. Molecular chaperones in protein folding and proteostasis. Nature 475:324-332.

Haslbeck, M., Franzmann, T., Weinfurtner, D., and Buchner, J. 2005. Some like it hot: The structure and function of small heat-shock proteins. Nat. Struct. Mol. Biol. 12:842-846.

Horsch, R. B., Fry, J. E., Hoffmann, N. L., Eichholtz, D., Rogers, S. G., and Fraley, R. T. 1985. A simple and general method for transferring genes into plants. Science 227:1229-1231.

Ishibashi, K., and Ishikawa, M. 2016. Replication of tobamovirus RNA. Annu. Rev. Phytopathol. 54:55-78.

Ishibashi, K., Masuda, K., Naito, S., Meshi, T., and Ishikawa, M. 2007. An inhibitor of viral RNA replication is encoded by a plant resistance gene. Proc. Natl. Acad. Sci. USA 104:13833-13838.

Ishibashi, K., Nishikiori, M., and Ishikawa, M. 2010. Interactions between tobamovirus replication proteins and cellular factors: Their impacts on virus multiplication. Mo. Plant-Microbe In. 23:1413-1419.

Jiang, S., Lu, Y., Li, K., Lin, L., Zheng, H., Yan, F., and Chen, J. 2014. Heat shock protein 70 is necessary for Rice stripe virus infection in plants. Mol. Plant Pathol. 15:907-917.

Kong, Q., Yuan, J., Gao, L., Zhao, S., Jiang, W., Huang, Y., and Bie, Z. 2014. Identification of suitable reference genes for gene expression normalization in qRT-PCR analysis in watermelon. PLoS One 9:e90612.

Lanneau, D., Wettstein, G., Bonniaud, P., and Garrido, C. 2010. Heat shock proteins: Cell protection through protein triage. Sci. World J. 10:15431552. 
Lee, G. J., Roseman, A. M., Saibil, H. R., and Vierling, E. 1997. A small heat shock protein stably binds heat-denatured model substrates and can maintain a substrate in a folding-competent state. EMBO J. 16:659-671.

Les Erickson, F., Holzberg, S., Calderon-Urrea, A., Handley, V., Axtell, M., Corr, C., and Baker, B. 1999. The helicase domain of the TMV replicase proteins induces the $\mathrm{N}$-mediated defense response in tobacco. Plant J. 18: 67-75.

Li, J., Xiang, C. Y., Yang, J., Chen, J. P., and Zhang, H. M. 2015. Interaction of HSP20 with a viral RdRp changes its sub-cellular localization and distribution pattern in plants. Sci. Rep. 5:14016.

Liang, H. H., Huang, C. Y., Chou, C. W., Makondi, P. T., Huang, M. T., Wei, P. L., and Chang, Y. J. 2018. Heat shock protein 27 influences the anticancer effect of curcumin in colon cancer cells through ROS production and autophagy activation. Life Sci. 209:43-51.

Lindquist, S., and Craig, E. A. 1988. The heat-shock proteins. Annu. Rev. Genet. 22:631-677.

Liu, L., Peng, B., Zhang, Z., Wu, Y., Miras, M., Aranda, M. A., and Gu, Q. 2017. Exploring different mutations at a single amino acid position of Cucumber green mottle mosaic virus replicase to attain stable symptom attenuation. Phytopathology 107:1080-1086.

Lu, L., Du, Z., Qin, M., Wang, P., Lan, H., Niu, X., Jia, D., Xie, L., Lin, Q., and Xie, L. 2009. Pc4, a putative movement protein of Rice stripe virus, interacts with a type I DnaJ protein and a small Hsp of rice. Virus Genes 38: 320-327.

Maimbo, M., Ohnishi, K., Hikichi, Y., Yoshioka, H., and Kiba, A. 2007. Induction of a small heat shock protein and its functional roles in Nicotiana plants in the defense response against Ralstonia solanacearum. Plant Physiol. 145:1588-1599.

Marshall, R. S., McLoughlin, F., and Vierstra, R. D. 2016. Autophagic turnover of inactive $26 \mathrm{~S}$ proteasomes in yeast is directed by the ubiquitin receptor Cue5 and the Hsp42 chaperone. Cell Reports 16:1717-1732.

Mchaourab, H. S., Godar, J. A., and Stewart, P. L. 2009. Structure and mechanism of protein stability sensors: The chaperone activity of small heat-shock proteins. Biochemistry 48:3828-3837.

Mine, A., Hyodo, K., Tajima, Y., Kusumanegara, K., Taniguchi, T., Kaido, M., Mise, K., Taniguchi, H., and Okuno, T. 2012. Differential roles of Hsp70 and Hsp90 in the assembly of the replicase complex of a positive-strand RNA plant virus. J. Virol. 86:12091-12104.

Nover, L., Scharf, K. D., and Neumann, D. 1989. Cytoplasmic heat shock granules are formed from precursor particles and are associated with a specific set of mRNAs. Mol. Cell. Biol. 9:1298-1308.

Padmanabhan, M. S., Goregaoker, S. P., Golem, S., Shiferaw, H., and Culver, J. N. 2005. Interaction of the tobacco mosaic virus replicase protein with the Aux/IAA protein PAP1/IAA26 is associated with disease development. J. Virol. 79:2549-2558.

Padmanabhan, M. S., Kramer, S. R., Wang, X., and Culver, J. N. 2008. Tobacco mosaic virus replicase-auxin/indole acetic acid protein interactions: Reprogramming the auxin response pathway to enhance virus infection. J. Virol. 82:2477-2485.
Piotrowska, J., Hansen, S. J., Park, N., Jamka, K., Sarnow, P., and Gustin, K. E. 2010. Stable formation of compositionally unique stress granules in virusinfected cells. J. Virol. 84:3654-3665.

Reingold, V., Lachman, O., Belausov, E., Koren, A., Mor, N., and Dombrovsky, A. 2016. Epidemiological study of Cucumber green mottle mosaic virus in greenhouses enables reduction of disease damage in cucurbit production. Ann. Appl. Biol. 168:29-40.

Rozanov, M. N., Koonin, E. V., and Gorbalenya, A. E. 1992. Conservation of the putative methyltransferase domain: A hallmark of the 'Sindbis-like' supergroup of positive-strand RNA viruses. J. Gen. Virol. 73:2129-2134.

Schmidt, G. W., and Delaney, S. K. 2010. Stable internal reference genes for normalization of real-time RT-PCR in tobacco (Nicotiana tabacum) during development and abiotic stress. Mol. Genet. Genomics 283:233-241.

Singleton, M. R., Dillingham, M. S., and Wigley, D. B. 2007. Structure and mechanism of helicases and nucleic acid translocases. Annu. Rev. Biochem. 76:23-50.

Sun, W., Van Montagu, M., and Verbruggen, N. 2002. Small heat shock proteins and stress tolerance in plants. BBA-Gen. Struct. Exp. 1577:1-9.

Tyedmers, J., Mogk, A., and Bukau, B. 2010. Cellular strategies for controlling protein aggregation. Nat. Rev. Mol. Cell Biol. 11:777-788.

Ueda, H., Yamaguchi, Y., and Sano, H. 2006. Direct interaction between the tobacco mosaic virus helicase domain and the ATP-bound resistance protein, $\mathrm{N}$ factor during the hypersensitive response in tobacco plants. Plant Mol. Biol. 61:31-45.

Ugaki, M., Tomiyama, M., Kakutani, T., Hidaka, S., Kiguchi, T., Nagata, R., Sato, T., Motoyoshi, F., and Nishiguchi, M. 1991. The complete nucleotide sequence of cucumber green mottle mosaic virus (SH strain) genomic RNA. J. Gen. Virol. 72:1487-1495.

Wang, W., Vinocur, B., Shoseyov, O., and Altman, A. 2004. Role of plant heatshock proteins and molecular chaperones in the abiotic stress response. Trends Plant Sci. 9:244-252.

Wang, X., Goregaoker, S. P., and Culver, J. N. 2009. Interaction of the Tobacco mosaic virus replicase protein with a NAC domain transcription factor is associated with the suppression of systemic host defenses. J. Virol. 83: 9720-9730.

Whitham, S. A., Quan, S., Chang, H. S., Cooper, B., Estes, B., Zhu, T., Wang, X., and Hou, Y. M. 2003. Diverse RNA viruses elicit the expression of common sets of genes in susceptible Arabidopsis thaliana plants. Plant J. 33:271-283.

Whitham, S. A., and Wang, Y. 2004. Roles for host factors in plant viral pathogenicity. Curr. Opin. Plant Biol. 7:365-371.

Whitley, D., Goldberg, S. P., and Jordan, W. D. 1999. Heat shock proteins: A review of the molecular chaperones. J. Vasc. Surg. 29:748-751.

Yamanaka, T., Imai, T., Satoh, R., Kawashima, A., Takahashi, M., Tomita, K., Kubota, K., Meshi, T., Naito, S., and Ishikawa, M. 2002. Complete inhibition of tobamovirus multiplication by simultaneous mutations in two homologous host genes. J. Virol. 76:2491-2497.

Zhang, K., Ezemaduka, A. N., Wang, Z., Hu, H., Shi, X., Liu, C., Lu, X. P., Fu, X. M., Chang, Z. Y., and Yin, C. C. 2015. A novel mechanism for small heat shock proteins to function as molecular chaperones. Sci. Rep. 5:8811. 